\title{
Correction Schemes to Improve the Dynamical Aperture of the Main Injector
}

\author{
C.S. Mishra and F.A. Harfoush \\ Fermi National Accelerator Laboratory \\ P.O. Box 500, Batavia, Illinois 60510
}

July 1993

Presented at the 1993 Particle Accelerator Conference, Washington, D.C., May 17-20, 1993 


\section{Disclaimer}

This report was prepared as an account of work sponsored by an agency of the United States Government. Neither the United States Government nor any agency thereof, nor any of their employees, makes any warranty, express or implied, or assumes any legal liability or responsibility for the accuracy, completeness, or usefulness of any information, apparatus, product, or process disclosed, or represents that its use would not infringe privately owned rights. Reference herein to any specific commercial product, process, or service by trade name, trademark, manufacturer, or otherwise, does not necessarily constitute or imply its endorsement, recommendation, or favoring by the United States Government or any agency thereof. The views and opinions of authors expressed herein do not necessarily state or reflect those of the United States Government or any agency thereof. 


\title{
Correction Schemes to Improve the Dynamical Aperture of the Main Injector
}

\author{
C. S. Mishra and F. A. Harfoush \\ Fermi National Accelerator Laboratory* \\ Batavia, Mlinois 60510
}

\begin{abstract}
Tracking simulations have revealed that the dominant factors limiting the dynamical aperture of the Main Injector are the large octupole component and the random errors in the recycled Main Ring quadrupoles. This paper describes a correction scheme that reduces the effect of these errors on the performance of the Fermilab Main Injector, especially for the slow extraction operation.
\end{abstract}

\section{INTRODUCTION}

The Fermilab Main Injector (FMI) is designed for multipurpose operation. At present four modes of operation for the FMI are planned. These correspond to antiproton production and slow or fast spill at $120 \mathrm{GeV} / \mathrm{c}$, fixed target and collider operation at $150 \mathrm{GeV} / \mathrm{c}$. Combinations of the above operational modes are also anticipated. It is important to understand the limitations of the lattice design and find new schemes which will improve the performance of the FMI. Simulations [1] of the FMI lattice, which include the magnetic field errors, both systematic and random, and misalignment errors, show that the dynamical aperture is larger than the design value of $40 \pi \mathrm{mm}$-mradians at injection. The dynamic aperture can become a limiting factor at slow extraction and therefore requires a closer study. Here the base tune is slowly increased to half integer resonance, before the extraction elements are turned on.

In this paper we discuss the limitations and correction schemes which utilizes the octupole and trim quadrupoles already placed in the lattice to increase the dynamic aperture.

\section{TRACKING CONDITIONS}

The FMI lattice has two different sizes of dipole magnets. For tracking calculations each dipole magnet is cut in three pieces, two ends and one body. The dipole body and end multipoles, both normal and skew, are calculated by using the method described in [2]. To this date only two prototype dipoles have been built, so the random body

\footnotetext{
*Operated by the Unjversities Research Association under contract with the U.S. Department of Energy
}

errors are calculated from the available measurements of the B2 dipoles in the Main Ring (MR). Systematic and random errors of the quadrupoles are calculated using the MR quadrupole measurements. These quadrupoles have a large octupole component and random error. The large random error in dipole and quadrupole field strength can be attributed to the limited number of available measurements. All skew quadrupole field errors are turned of for the convenience of the simulation. Tables 1 and 2 of [2] summarize all of the multipoles used in the input file to the thin element tracking program TEAPOT [3].

The misalignment of all the magnetic elements and beam position monitors has been included in our calculations. The rms of the alignment error with respect to the closed orbit is $0.25 \mathrm{~mm}$ in both horizontal and vertical planes. In addition dipole magnets have an rms roll angle of 0.5 mrad.

Base tune of FMI is $\left(Q_{x}, Q_{y}\right)=(26.425,25.415)$. This base tune has been used in our studies of the dynamic aperture except at slow extraction where the horizontal tune is changed to 26.485. This is further discussed in this paper. In the lattice there are $18 \mathrm{RF}$ cavities, each operating at $V_{\mathrm{r} f}=0.0218 \mathrm{MV}$ and $0.0555 \mathrm{MV}$ at 8.9 and $120 \mathrm{GeV}$ respectively. The RF frequency is set to $53 \mathrm{MHz}$ corresponding to a harmonic number of 588 . Synchrotron oscillation was included in the simulations by launching all particles with an amplitude of $\delta_{\text {max }}=(\Delta p / p)_{\max }=$ $2.0 \mathrm{E}-3,0.3 \mathrm{E}-3$ at 8.9 and $120.0 \mathrm{GeV}$ respectively.

\section{TRACKING RESULTS}

We have studied the variation of the horizontal and vertical tunes as the amplitude of motion is increased. A particle is launched with a maximum horizontal displacement of " $A$ " at a location where the horizontal beta function is at its maximum of 75 meters. The maximum vertical displacement of the same particle is $0.4 \mathrm{~A}(\mathrm{x} / \mathrm{y}=2.5)$ also at beta of 75 meters. Synchroton oscillation is included in the simulation by launching the particles with the proper non zero $\Delta p / p$. Figs $1 \mathrm{a}$ and $1 \mathrm{~b}$ show the tune-tune plot at 8.9 and $120 \mathrm{GeV} / \mathrm{c}$ respectively. The particle labels on the tune plot correspond to the initial amplitude " $\mathrm{A}$ " of a test particle in millimeters. Plot of tune versus amplitude is very close to a straight line for amplitudes less than 28 
$\mathrm{mm}$ at $8.9 \mathrm{GeV} / \mathrm{c}$, and less than $31 \mathrm{~mm}$ at $120 \mathrm{GeV} / \mathrm{c}$. The nonlinear detuning at larger amplitudes is dominated by a combination of systematic octupole error in the recycled MR quadrupoles, and second order sextupole effects. Half of this detuning is due to the octupole component.

The presence of the dipole and quadrupole random errors and magnet alignment errors contributes to a variation of the $\beta$ function around the FMI away from the error free lattice, as discussed in [1]. The average $\Delta \beta / \beta_{\max }$ is about $6.4 \%$. Fig. $2 \mathrm{a}$ is sample $\beta$ function variation around the ring at a given seed with all the errors described earlier included.

The dynamical aperture of the FMI at $8.9 \mathrm{GeV}$ and 120 $\mathrm{GeV}$ has been discussed in [1]. At $120 \mathrm{GeV}$, close to the half integer resonance extraction, the horizontal tune is changed from $Q_{x}=26.425$ to 26.485 . This is achieved by ramping the main quadrupoles in $0.1 \mathrm{sec}$. The detuning of the particle will remain the same at this setting and will result in loss of large amplitude particle. The slow extraction septum is placed at $16 \mathrm{~mm}$. We have studied the dynamical aperture of the FMI for slow extraction. In this regime particles remain in the FMI for $1.0 \mathrm{sec}$ equivalent to about 100k turns. A survival plot is shown in Fig. 3. The dynamical aperture at $120 \mathrm{GeV}$ is reduced from $34.6 \pm 0.5$ to $20.67 \pm 1.25 \mathrm{~mm}$. These calculations have not taken into account other effects like power supply ripple, and space charge. If included we expect a further reduction in the dynamical aperture. Because the beam behaviour is more unstable at slow extraction a bigger dynamical aperture is needed.

\section{IMPROVEMENTS}

The focusing and defocusing quadrupoles are powered by two separate buses, and the current in each bus can be different. All the recycled MR quadrupoles will be measured for field quality and retuned if necessary before they are placed in the FMI. A smart shuffling scheme can be worked out that reduces the rms error in quadrupole field strength around the ring. A simple scheme is to divide the quadrupoles into two groups. One group will contain the quadrupoles with a strength higher than the total mean value and another group will contain the lower strength. This separation into two groups will reduce the rms error of quadrupoles by creating two non gaussian distributions. This has the equivalent effect of dividing the rms error by $2 \sqrt{2}$. To simulate this process in our tracking we have reduced the rms error of quadrupoles from $24.0 \mathrm{E}-4\left(1 / \mathrm{in}^{2}\right)$ to $8.0 \mathrm{E}-4\left(1 / \mathrm{in}^{2}\right)$.

The FMI lattice has 32 octupoles and 16 trim quadrupoles placed around the ring for extraction. There are also 8 corrector octupoles in the ring. We are evaluating the possibilities of adding 8 more similar octupoles. The MR quadrupoles have a positive octupole component almost invariant with energy. The extraction and corrector octupoles will be used to zero the total octupole component in the ring. The bipolar power supply on these mag- nets will enable us to use them for slow extraction, and as correctors at all other energies.

Fig. 4 shows the detuning of the particles at $120 \mathrm{GeV}$ with the quadrupole random error reduced by $\frac{1}{3}$, and with the octupole correction elements turned on. Detuning of particles with large amplitude is reduced by about $30 \%$. Fig. 5, is the survival plot before and after correction for one seed at the slow extraction tune of $\left(Q_{x}, Q_{y}\right)$ $=(26.485,25.425)$. The dynamical aperture increases by about $7 \mathrm{~mm}$. This is due mainly to the reduced $\Delta \beta / \beta$, and to the smaller total octupole in the FMI.

The variation in $\Delta \beta / \beta$ can be further reduced by canceling the natural half-integer stopband of the FMI. This is achieved by using the trim quadrupoles placed in the ring for slow extraction. Just like octupoles these trim quadrupoles can be used as extraction element for slow extraction, and as correctors at all other energies. Fig. 2 (b) shows the $\Delta \beta / \beta$ of the FMI after the quadrupole random error, octupole, and half-integer stopband corrections have been included. Fig. 5, also shows that the dynamical aperture does not increase when the half-integer stop band correction is added to the two other corrections.

\section{CONCLUSION}

Using the correction schemes described in this paper our studies have shown that it is possible to reduce the effect of the quadrupole random error, and octupole multipole. This correction scheme provides us with additional aperture at all energies, especially for the $120 \mathrm{GeV}$ slow extraction.

\section{ACKNOWLEDGMENTS}

We thank R. Talman for his help with the tracking code TEAPOT. We also thank Phil Martin and Steve Peggs for the discussions throughout this study.

\section{REFERENCES}

[1] C. S. Mishra and F. A. Harfoush, "Simulation of the Dynamical Performance of the Main Injector," Fermi Internal Notes MI-0070, FERMILAB-CONF-92/075 and in PAC93 proceedings.

[2] F. A. Harfoush and C. S. Mishra, "Systematic and Random Errors for Main Injector Tracking," Fermi Internal Notes, MI-0066, in PAC93 proceedings.

[3] L. Schachinger and R. Talman, Particle Accl. 22, $35(1987)$. 


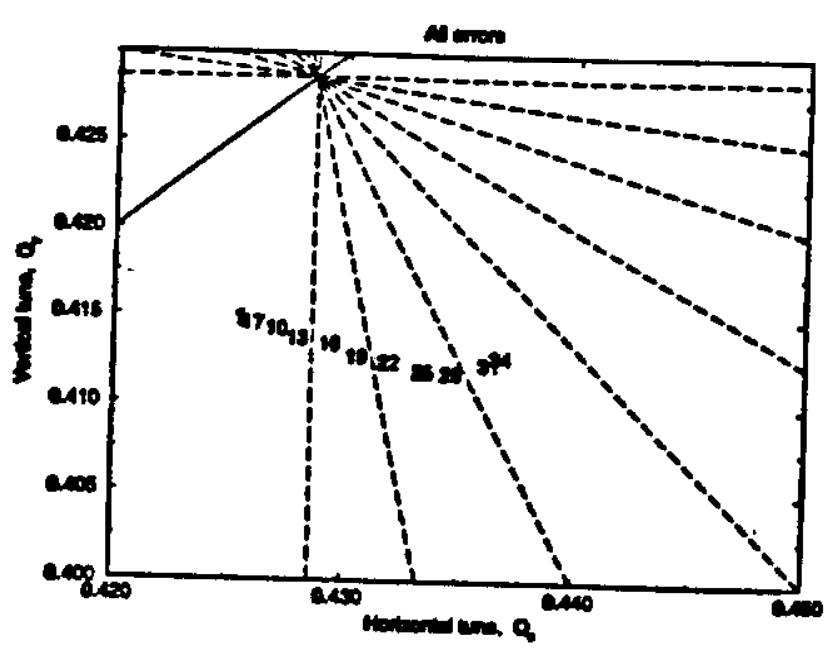

Fig. 1a Tune Tune plot for $8.0 \mathrm{GoV}$

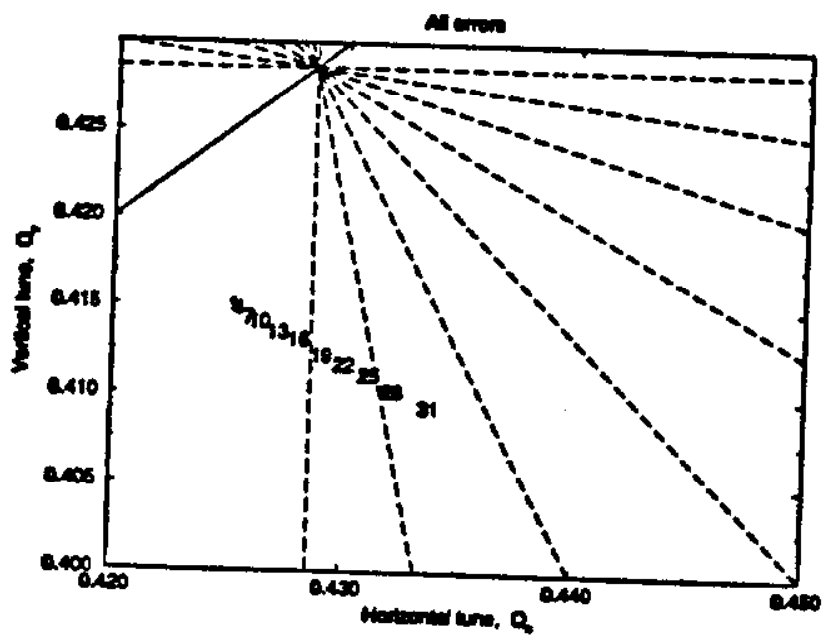

Fig. 16 Tune Tune plot for $120.0 \mathrm{GoV}$

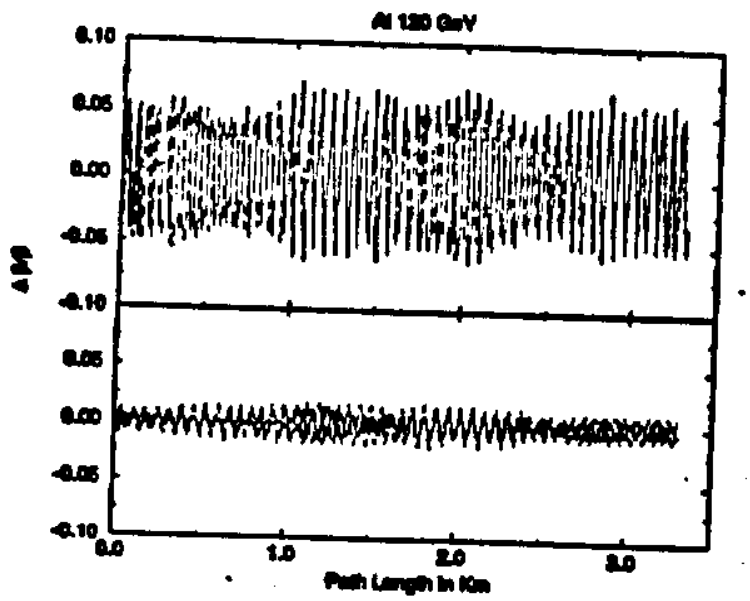

Fig. 2a Variation in Beta Function

Fig. 2b Variation in Beta Function with corriction:

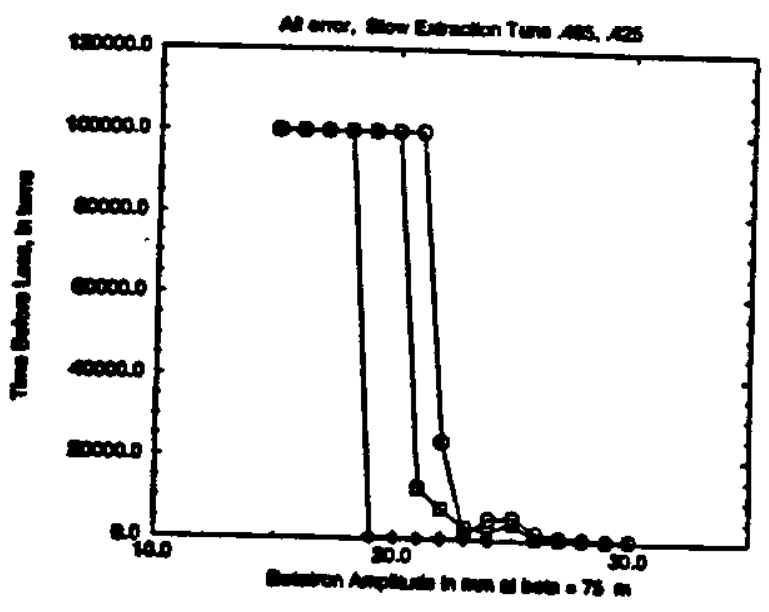

Fig. 3. Survival Plot at 120. Cov

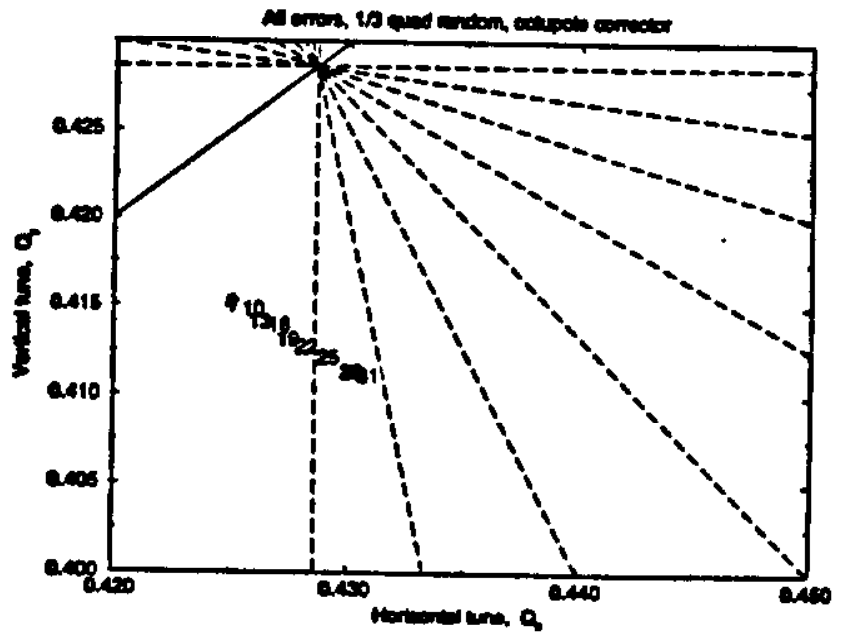

Fig. 4 Tune Tune plot for $120.0 \mathrm{GeV}$

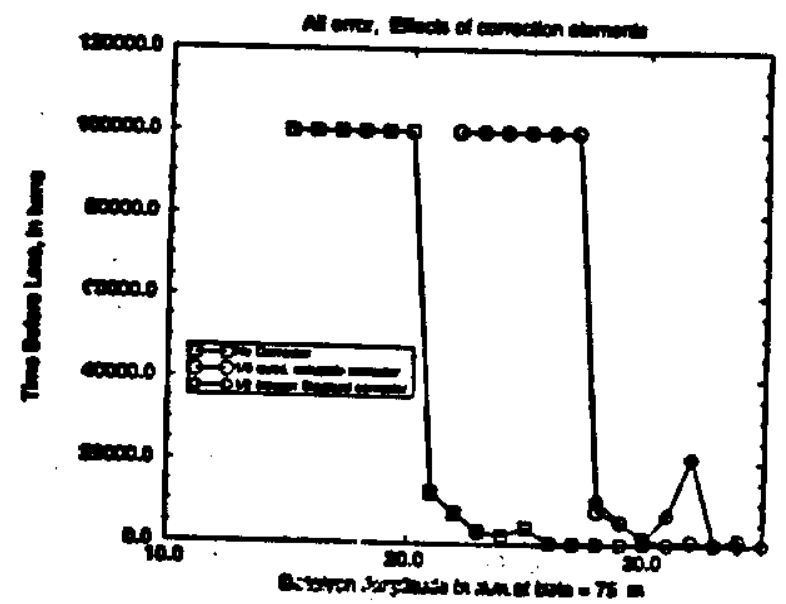

Fo. 5 Survival Plot at 120. GoV 